\title{
Resveratrol relieves ischemia-induced oxidative stress in the hippocampus by activating SIRT1
}

\author{
ZHUANGZHI MENG ${ }^{1,2^{*}}$, JIANGUO LI ${ }^{1-3^{*}}$, HONGLIN ZHAO ${ }^{1,2^{*}}$, HAIYING LIU ${ }^{1,2}$, GUOWEI ZHANG ${ }^{1,2}$, \\ LINGZHAN WANG ${ }^{1,2}$, HE HU ${ }^{1,2}$, DI LI ${ }^{1,2}$, MINGJING LIU ${ }^{1,2}$, FULONG BI ${ }^{1,2}$, XIAOPING WANG ${ }^{1,2}$, \\ GENG TIAN $^{1,2}$, QIANG LIU ${ }^{4}$ and BATU BUREN ${ }^{3}$
}

\begin{abstract}
Departments of ${ }^{1}$ Human Anatomy and ${ }^{2}$ Preventive Medicine, The School of Medicine of Inner Mongolia University for The Nationalities, Tongliao, Inner Mongolia 028041; ${ }^{3}$ Laboratory of Biomedicine and Department of Mongolian Medicine

Hematology-Oncology, The Affiliated Hospital of Inner Mongolia University for The Nationalities, Tongliao, Inner Mongolia 028007; ${ }^{4}$ Tianjin Key Laboratory of Molecular Nuclear Medicine, Institute of Radiation Medicine, Chinese Academy of Medical Science and Peking Union Medical College, Tianjin 300192, P.R. China
\end{abstract}

Received August 5, 2014; Accepted April 27, 2015

DOI: 10.3892/etm.2015.2555

\begin{abstract}
Resveratrol, a naturally occurring phytoalexin, acts as an activator of sirtuin 1 (SIRT1) and has been shown to have a neuroprotective role in various models. Healthy adult male Sprague-Dawley rats were subjected to cerebral ischemia in order to study the protective effect of resveratrol on the brain following ischemia, and to investigate the effects of SIRT1 activation on the hippocampus. Untreated and resveratrol-treated rats were anesthetized prior to undergoing surgery to induce middle cerebral artery occlusion followed by reperfusion. SIRT1 expression was evaluated by immunohistochemistry, western blotting and reverse transcription-quantitative polymerase chain reaction, and SIRT1 activity was also evaluated. In addition, terminal deoxynucleotidyl transferase dUTP nick end-labeling (TUNEL) and Nissl staining assays were conducted and the levels of reactive oxygen species were determined. It was observed that resveratrol significantly decreased the number of TUNEL-positive cells and increased the expression of SIRT1 mRNA in a dose-dependent manner.
\end{abstract}

Correspondence to: Dr Qiang Liu, Tianjin Key Laboratory of Molecular Nuclear Medicine, Institute of Radiation Medicine, Chinese Academy of Medical Sciences and Peking Union Medical College, 238 Baidi Road, Tianjin 300192, P.R. China

E-mail: liuqiang70@126.com

Dr Batu Buren, Laboratory of Biomedicine and Department of Mongolian Medicine Hematology-Oncology, The Affiliated Hospital of Inner Mongolia University for The Nationalities, 1742 Huolinhe Street, Tongliao, Inner Mongolia 028007, P.R. China E-mail: burenbatutlmd@126.com

*Contributed equally

Key words: resveratrol, sirtuin 1, brain ischemia, reactive oxygen species, hippocampus
This was accompanied by increases in SIRT1 protein expression levels and SIRT1 activity. The results demonstrate the neuroprotective and antioxidant effects of resveratrol against ischemia-induced apoptosis in the rat hippocampus.

\section{Introduction}

Stroke has been recognized as a leading cause of disability and is the second most common cause of mortality in adults worldwide (1). Research has indicated that the use of neuroprotective agents may be a suitable approach to the treatment of stroke. Although numerous clinical trials have been conducted with potentially neuroprotective agents, few have been used clinically $(2,3)$.

During ischemic insult, the production of reactive oxygen species (ROS) increases. This leads to neuronal death, brain edema and inflammation (4). ROS produced at the early phase of neuronal apoptosis act as mediators of intracellular apoptotic signaling cascades (5), and pathologically accelerate the release of cytochrome $c$ from mitochondria $(6,7)$. ROS production evidently increases following ischemia, which leads to oxidative stress (8-10). Studies have revealed that at least some of the effects on cells of exposure to ROS are similar to those of ischemic insult. The brain, which is a major metabolic organ of oxygen and has relatively weak protective antioxidant mechanisms, is particularly vulnerable to the insult perpetrated by ROS. The neuroprotective role of sirtuin 1 (SIRT1) has been demonstrated in models of various neurodegenerative diseases, including Alzheimer's disease, Parkinson's disease and Huntington's disease $(11,12)$.

Resveratrol (RSV; 3,5,4'-trihydroxy-trans-stilbene) is a naturally occurring phytoalexin that is present in the skin of red grapes and is a component of red wine $(13,14)$. It mediates a variety of biological activities that are associated with extension of life span, even in those with a high caloric diet, and cancer prevention $(15,16)$. Resveratrol acts as an activator of SIRT1 and has been shown to be neuroprotective in various models (16-18). The hippocampus is a region of active prolif- 
eration and neurogenesis within the brain. Ischemia induces apoptosis of neurons in the subventricular zone of the lateral ventricles and in the subgranular zone of the hippocampus in the adult brain. Transient global ischemia induces neuronal damage specifically in the hippocampus of rats $(19,20)$.

In the current study, the aim was to investigate whether resveratrol inhibits the caspase cell death pathway to protect hippocampal neurons from ischemia and evaluate the effects of SIRT1 activation in the hippocampus. The mechanisms that lead to this phenomenon within neurons were also investigated.

\section{Materials and methods}

Chemicals and animals. Resveratrol was obtained from Sigma Chemicals (St. Louis, MO, USA). Male Sprague-Dawley rats weighing 200-220 $\mathrm{g}(\mathrm{n}=24)$ were obtained from the Experimental Animal Research Center, Institute of Radiation Medicine, Chinese Academy of Medical Sciences and Peking Union Medical College, Tianjin, China. The rats were maintained in a room at $22 \pm 2^{\circ} \mathrm{C}$, with a relative humidity of $50 \pm 10 \%$, on a $12 \mathrm{~h}$ light-dark cycle. All animal experiments were conducted in accordance with a protocol approved by the Institutional Animal Care and Use Committee (IACUC) of the Institute of Radiation Medicine, Chinese Academy of Medical Sciences. (No. 1204). Rats were randomly divided into four groups (6 rats/group). These were the ischemia group (ISC group), the ischemia with $5 \mathrm{mg} / \mathrm{kg}$ resveratrol group (ISC + RSV-5 group), the ischemia with $10 \mathrm{mg} / \mathrm{kg}$ resveratrol group (ISC + RSV-10 group) and the control group.

Drug treatments. Rats in the control and ISC groups were given distilled water, while the rats in the ISC + RSV-5 and ISC + RSV-10 groups were respectively given drinking water plus 5 and $10 \mathrm{mg} / \mathrm{kg}$ resveratrol for 21 days.

Middle cerebral artery occlusion. Rats in the ISC group and the two ISC + RSV groups were anesthetized with $2 \%$ Napental (4 mg/kg, intraperitoneally) and then underwent surgery to induce middle cerebral artery occlusion. The middle cerebral artery was ligated with an intraluminal filament for $1.5 \mathrm{~h}$ and then reperfused for $24 \mathrm{~h}$. For the sham group, the external carotid artery was surgically prepared for insertion of the filament, but no filament was inserted. During the experimental procedures, blood pressure, blood gas level and glucose levels were monitored. Rectal temperature was maintained at $37.0-37.5^{\circ} \mathrm{C}$ with a heating pad, and body temperature was maintained at $37^{\circ} \mathrm{C}$ with a thermostatically controlled infrared lamp. Brain temperature was maintained at $36-37^{\circ} \mathrm{C}$ and monitored with a 29 -gauge thermocouple in the right corpus striatum and a temperature-regulating lamp. An electroencephalogram was taken to ensure isoelectricity during the ischemic period (19).

Tissue preparation. At $24 \mathrm{~h}$ after the experimental interventions, the rats were deeply anesthetized, sacrificed by an overdose of anesthesia and quickly treated with a cardiac perfusion of $4 \%$ paraformaldehyde. The hippocampi from 3 rats per group were extracted and cut into $12-\mu \mathrm{m}$ coronal sections with a cryostat (CM 3000; Leica, Manheim, Germany) then placed on glass slides and stored at $-80^{\circ} \mathrm{C}(21,22)$.
Immunohistology, terminal deoxynucleotidyl transferase dUTP nick end-labeling (TUNEL) staining and cresyl violet $(C V)$ staining. The avidin-biotin-peroxidase complex method of immunohistochemical staining was conducted as previously described (23). DNA fragmentation was detected using a TUNEL kit (Roche Diagnostics Corporation, Indianapolis, IN, USA) according to the manufacturer's instructions. The sections were stained with cresyl violet (CV) using the conventional method and mounted (24).

Western blot analysis. The total protein and nuclear protein were isolated from hippocampi using RIPA buffer (Beyotime, Jiangsu, China) according to the manufacturer's instructions. The protein concentration of the supernatant homogenate was determined using a Bio-Rad kit (Bio-Rad, Hercules, CA, USA) at an absorbance of $595 \mathrm{~nm}$. The samples $(80 \mu \mathrm{g})$ were transferred to polyvinylidene difluoride membranes and incubated with the following primary antibodies: Rabbit monoclonal anti-SIRT1 antibodies obtained from Cell Signaling Technology (dilution 1:500; cat. no. 9475P, Beverly, MA, USA); rabbit monoclonal anti- $\beta$-actin (dilution 1:1,500; Sangon Biotech, Shanghai, China) was used for loading control and the secondary antibody was goat anti-rabbit IgG conjugated to horseradish peroxidase (dilution 1:500; ZSGB-Bio, Beijing, China).

RNA extraction, cDNA synthesis and reverse transcription-quantitative polymerase chain reaction ( $R T$-qPCR). Total RNA was purified and extracted as described previously by Chen et al (25). Equal concentrations of total RNA were reverse-transcribed using Prime Script RT reagent kit (Takara Bio Inc., Shiga, Japan) according to the manufacturer's instructions. cDNA samples were blended with primers and SYBR Master Mix (Invitrogen Life Technologies, Carlsbad, CA, USA) in a total volume of $25 \mathrm{ml}$. The samples were assayed in triplicate using an ABI PRISM ${ }^{\circledR} 7500$ Sequence Detection system (Applied Biosystems-Life Technologies, Foster City, CA, USA). The cycle threshold (CT) values for each reaction were determined and the mean was calculated using TaqMan SDS analysis software (Applied Biosystems-Life Technologies). The expression levels of target genes were determined by the comparative CT method (fold change $=2^{-\Delta \Delta C T}$ ). PCR primers for SIRT1 were obtained from Sangon Biotech (Shanghai, China). The primer pairs for SIRT1 were as follows: forward, 5'-CCAGATCCTCAAGCCATGT-3' and reverse, 5'-TTGGATTCCTGCAACCTG-3' (26).

Analysis of reactive oxygen species and SIRT1 activity. The fluorogenic probe 2',7'-dichlorodihydrofluorescein diacetate was used to assess the production of ROS, as previously described (22). The activity of SIRT1 was analyzed using a fluorogenic assay according to the technique previously described by Li et al (22).

Statistical analysis. Data are presented as the mean \pm standard deviation. Data were analyzed using one-way analysis of variance with a post hoc test (multiple comparison test), which determined the significant differences among groups. $\mathrm{P}<0.05$ was considered to indicate a statistically significant difference. 


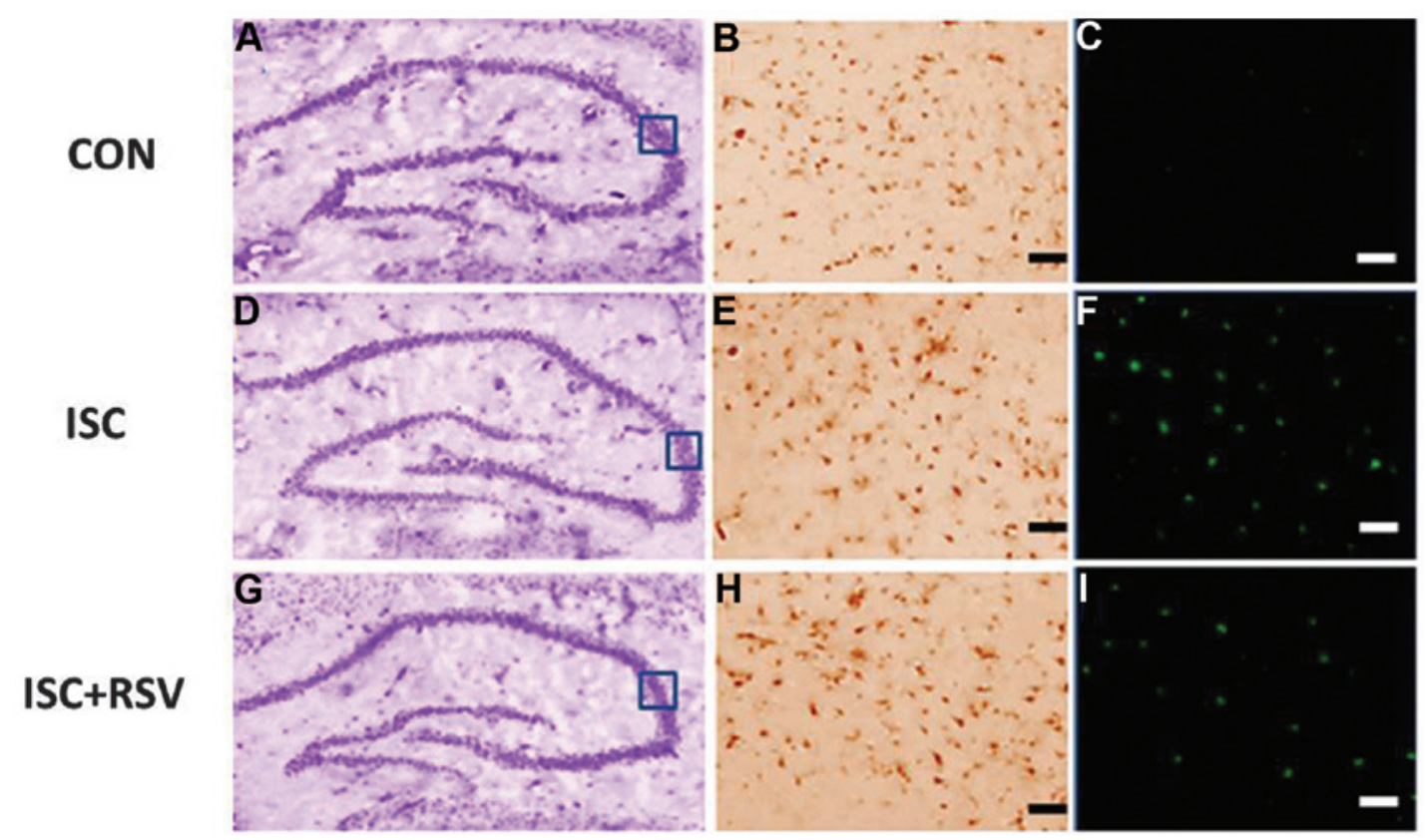

Figure 1. Representative images for (A, D, G) CV, (B, E, H) SIRT1 and (C, F, I) TUNEL staining in the CA3 hippocampal region. Scale bars: (B, C, E, F, $\mathrm{H}, \mathrm{I}) 50 \mu \mathrm{m}$. The box indicates the image positioning of SIRT1 and TUNEL. CV, cresyl violet; TUNEL, terminal deoxynucleotidyl transferase dUTP nick end-labeling; SIRT1, sirtuin 1; CON, control; ISC, ischemia; RSV, resveratrol-10.

A

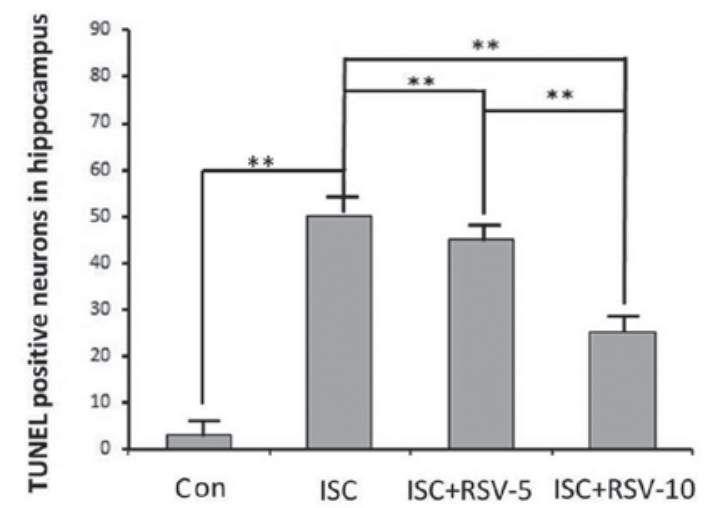

B

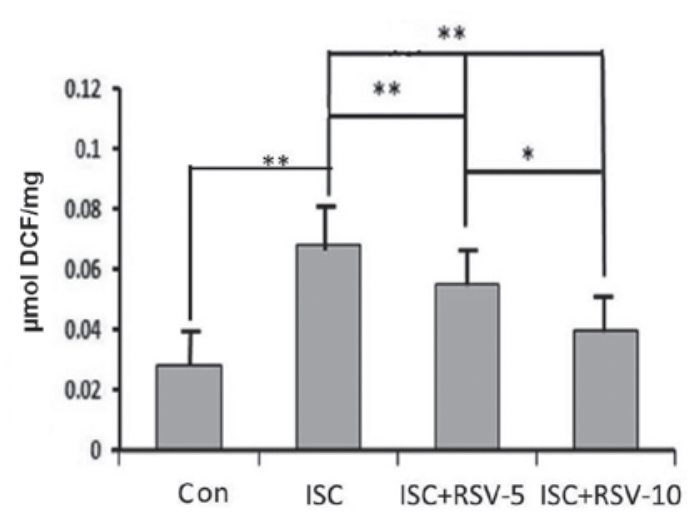

Figure 2. (A) TUNEL staining results and (B) mean values of ROS production. TUNEL, terminal deoxynucleotidyl transferase dUTP nick end-labeling; ROS, reactive oxygen species; DCF, dichlorofluorescein; Con, control; ISC, ischemia; RSV-5, $5 \mathrm{mg} / \mathrm{kg}$ resveratrol; RSV-10, $10 \mathrm{mg} / \mathrm{kg}$ resveratrol. ${ }^{*} \mathrm{P}<0.05,{ }^{* *} \mathrm{P}<0.01$.

A

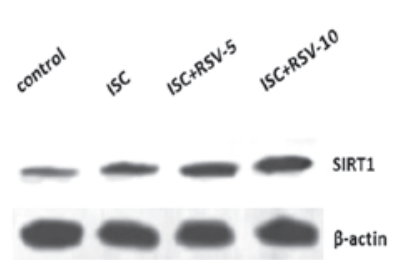

B

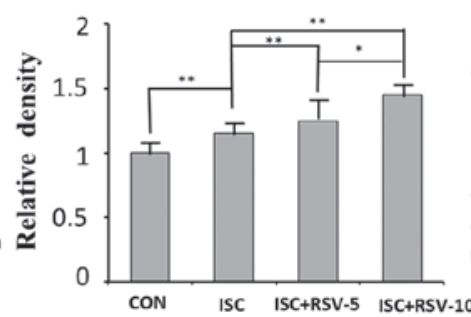

C

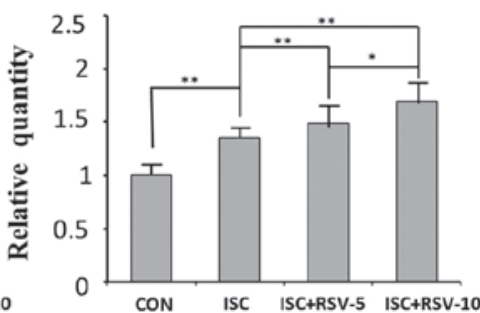

Figure 3. Effects of resveratrol on the expression levels of SIRT1. (A) Western blotting results, (B) protein expression levels and (C) mRNA expression levels (expressed as relative values, which are mean \pm standard deviation values compared with the control). SIRT1, sirtuin 1; Con, control; ISC, ischemia; RSV-5, $5 \mathrm{mg} / \mathrm{kg}$ resveratrol; RSV-10, $10 \mathrm{mg} / \mathrm{kg}$ resveratrol. ${ }^{*} \mathrm{P}<0.05,{ }^{* *} \mathrm{P}<0.01$.

\section{Results}

Immunohistology and TUNEL-positive cells within the hippocampi. CV staining was conducted to stain Nissl bodies a purple-blue color (Fig. 1A, D and G). This revealed that distinct damage of neurons occurred in the ISC and ISC + RSV-10 groups (Fig. 1D and G) when compared with the control group (Fig. 1A). Immunohistochemical staining 


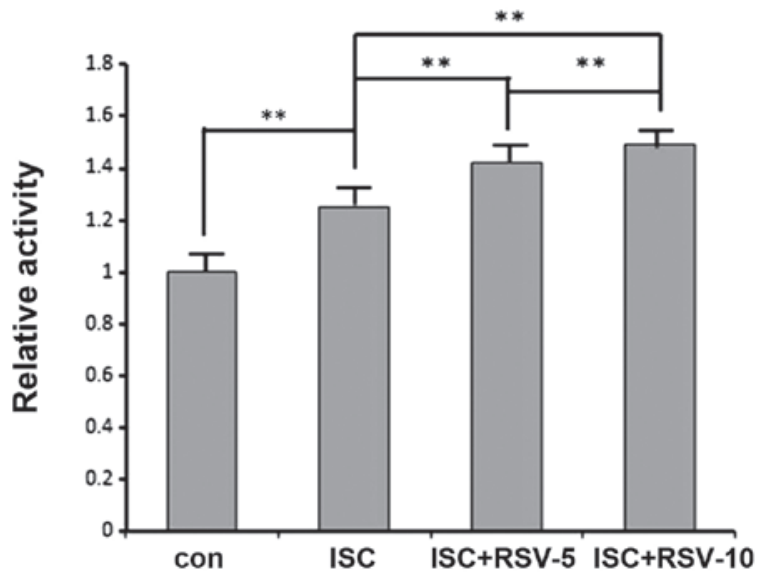

Figure 4. SIRT1 enzyme activity in rats following ischemia (ISC) and resveratrol treatment, measured in total protein extracts from the hippocampal tissues. Results were normalized to control levels. SIRT1, sirtuin 1; RSV-5, 5 $\mathrm{mg} / \mathrm{kg}$ resveratrol; RSV-10, $10 \mathrm{mg} / \mathrm{kg}$ resveratrol. ${ }^{* *} \mathrm{P}<0.01$.

of SIRT1 in the hippocampi of the ISC group (Fig. 1H) was more intense than in the ISC + RSV group (Fig. 1E), which in turn was more intense than that in the control group (Fig. 1B). TUNEL-positive cells were most visible in the hippocampi of the ISC group (Fig. 1F), and visible at lower levels in the ISC + RSV group (Fig. 1I). The number of TUNEL-positive cells detected in the control rats was low (Fig. 1C) compared with that in the other two groups. These results indicate that RSV attenuated the ischemia-induced damage. The neurons in the hippocampi from control rats displayed few positive neurons. Rats in the ISC group notably became evident by a prominent growth in the number of TUNEL positive cells. TUNEL positive neurons in the ISC+RSV group markedly decreased compared with ischemia only (Fig. 2A).

ROS accumulation. As shown in Fig. 2B, ischemia increased the levels of ROS that were detected in the rat hippocampi. In addition, RSV effectively attenuated the ischemia-induced ROS production in the RSV-treated groups in a dose-dependent manner.

SIRT1 expression determined by western blotting and RT-qPCR analysis and SIRT1 activity. The expression levels of SIRT1 in the ISC and ISC + RSV groups exhibited significant differences compared with those in the control group at the protein and mRNA levels (Fig. 3). Treatment with resveratrol increased the expression of SIRT1 in a concentration-dependent manner (Fig. 3). SIRT1 activity was significantly increased following ischemia, and was particularly high in the ISC + RSV-5 and ISC + RSV-10 groups, where resveratrol increased the SIRT1 activity in a concentration-dependent manner (Fig. 4).

\section{Discussion}

The results of the present study revealed that SIRT1 was highly expressed in rat hippocampal neurons following ischemia and that resveratrol effectively antagonized the oxidation induced by ischemia. SIRT1 provides neurons with tolerance against oxidative stress. It also plays an important role in the regulation of cellular functions. It can act on numerous non-histone proteins, including transcription factors and transcriptional coregulatory proteins $(11,13)$. SIRT1 participates in the control of systemic metabolism via the regulation of glucose and lipid homeostasis by deacetylating various targets, and also increases chromatin silencing. Various target proteins are substrates for SIRT1 (27). Histone acetylation is closely associated with active transcription, and histone methylation is often associated with transcriptional repression (28). SIRT1 suppresses the expression of inflammatory genes by enhancing the activities of histone methyl transferases. For example, SIRT1 deacetylates and activates histone methyl transferase, resulting in increased levels of trimethyl-substituted histone, which suppresses the expression of inducible inflammatory genes (29).

Previous studies have suggested that SIRT1 activation elicits resistance to oxidative stress through the regulation of transcription factors and coactivators such as Hif-2a and NF- $\mathrm{B}$ (30). P53 is an important factor involved in myocardial apoptosis, whose effects are achieved through the activation of the renin-angiotensin system. A study has indicated that when myocardial ischemia occurs, SIRT1 reduces the activity of P53 through deacetylation (31). SIRT1 is ubiquitously expressed in all tissues, particularly in the brain, and has been demonstrated to be a nuclear protein (32). However, SIRT1 has both nuclear import and export sequences and has been found to be present in the cytosolic fraction of mouse brains (33). Oxidative stress occurs as the result of a shift in balance that favors the generation of oxygen-derived ROS over certain antioxidant defense mechanisms. ROS can cause lipid peroxidation and lead to a loss of membrane integrity, reduction of mitochondrial membrane potential and increased permeability to $\mathrm{Ca}^{2+}$ in the plasma membrane (34). In the central nervous system, neurons are particularly vulnerable to assault by neurotoxins. Ischemia and oxidative stress are common underlying factors in this type of damage. In addition to exerting direct effects on vascular tone, ROS also impair vasomotor responses to other stimuli. Resveratrol has been identified to have notable antiinflammatory activity. It downregulates the activation of immune cells and the subsequent synthesis and release of pro-inflammatory mediators through the inhibition of transcriptional factors such as NF- $\kappa \mathrm{B}$. Resveratrol has also been shown to inhibit the activation of microglia $(35,36)$.

In a number of experimental paradigms, resveratrol is used to increase SIRT1 activity. However, recent studies $(26,37,38)$ have demonstrated that resveratrol does not directly activate SIRT1, which indicates that mediators may be involved in the activation process. Certain studies, discussed in a review (38), support the hypothesis that mechanisms other than direct activation bring about P53 acetylation by resveratrol. Another study demonstrated that SIRT1-dependent pathways, in addition to nitric oxide (NO)-dependent mechanisms, contribute to the beneficial mitochondrial and cellular effects of dietary restriction (39). Many of the effects that have been observed in resveratrol-treated animals are consistent with the modulation of SIRT1 targets (40). SIRT1 may regulate a number of pathways involved in mitochondrial biogenesis. The pathways controlled by endothelium-derived NO are suggested to be the most important (41). Resveratrol improves 
NO production by upregulating endothelial NO synthase at the level of transcription (42). However, whether resveratrol modulates other critical genes remains unclear. Although SIRT1 levels have been observed to increase following the administration of resveratrol, the protective effect of resveratrol may be SIRT1-independent. Future studies to clarify the understanding of the cellular mechanisms involved in the neuroprotective effects of resveratrol may provide new avenues for the treatment of ischemia-induced disorders and experiments using SIRT1-knockout rats may reveal the true correlation between SIRT1 and resveratrol.

In conclusion, in the present study, it was observed that resveratrol significantly decreased the number of TUNEL-positive cells, and increased SIRT1 mRNA expression levels, in addition to increasing the expression levels of SIRT1 protein and SIRT1 activity. The results indicate the neuroprotective and antioxidant effects of resveratrol against ischemia-induced apoptosis in the rat hippocampus.

\section{Acknowledgements}

The authors are grateful to the National Science \& Technology Pillar Program during the 12th Five-year Plan Period (No. 2012BA127B02) and the PUMC graduate student innovation fund.

\section{References}

1. Feigin VL, Lawes CM, Bennett DA and Anderson CS: Stroke epidemiology: A review of population-based studies of incidence, prevalence and case-fatality in the late 20th century. Lancet Neurol 2: 43-53, 2003.

2. Green AR, Odergren T and Ashwood T: Animal models of stroke: Do they have value for discovering neuroprotective agents? Trend Pharmacol Sci 24: 402-408, 2003.

3. Xu SY and Pan SY: The failure of animal models of neuroprotection in acute ischemic stroke to translate to clinical efficacy. Med Sci Monit Basic Res 19: 37-45, 2013.

4. Moro MA, Almeida A, Bolanos JP and Lizasoain I: Mitochondrial respiratory chain and free radical generation in stroke. Free Radic Biol Med 39: 1291-1304, 2005.

5. Martin-Romero FJ, Garcia-Martin E and Gutierrez-Merino C: Inhibition of oxidative stress produced by plasma membrane NADH oxidase delays low-potassium-induced apoptosis of cerebellar granule cells. J Neurochem 82: 705-715, 2002.

6. Atlante A, Bobba A, Calissano P, Passarella S and Marra E: The apoptosis/necrosis transition in cerebellar granule cells depends on the mutual relationship of the antioxidant and the proteolytic systems which regulate ROS production and cytochrome $c$ release enroute to death. J Neurochem 84: 960-971, 2003.

7. Li J, Wang Y, DU L, Xu C, Cao J, Wang Q, Liu Q and Fan F: Radiation-induced cytochrome $c$ release and the neuroprotective effects of the pan-caspase inhibitor z-VAD-fmk in the hypoglossal nucleus. Exp Ther Med 7: 383-388, 2014.

8. Kleikers PW, Wingler K, Hermans JJ, Diebold I, Altenhöfer S, Radermacher KA, Janssen B, Görlach A and Schmidt HH: NADPH oxidases as a source of oxidative stress and molecular target in ischemia/reperfusion injury. J Mol Med (Berl) 90: 1391-1406, 2012.

9. Zuo L and Motherwell MS: The impact of reactive oxygen species and genetic mitochondrial mutations in Parkinson's disease. Gene 532: 18-23, 2013.

10. Sochocka M, Koutsouraki ES, Gasiorowski K and Leszek J: Vascular oxidative stress and mitochondrial failure in the pathobiology of Alzheimer's disease: A new approach to therapy. CNS Neurol Disord Drug Targets 12: 870-881, 2013.

11. Jeong H, Cohen DE, Cui L, Supinski A, Savas JN, Mazzulli JR, Yates JR III, Bordone L, Guarente L and Krainc D: SIRT1 mediates neuroprotection from mutant huntingtin by activation of the TORC1 and CREB transcriptional pathway. Nat Med 18: $159-165,2011$.
12. Donmez G, Arun A, Chung CY, McLean PJ, Lindquist S and Guarente L: SIRT1 protects against alpha-synuclein aggregation by activating molecular chaperones. J Neurosci 32: 124-132, 2012.

13. Chong ZZ, Shang YC, Wang S and Maiese K: SIRT1: New avenues of discovery for disorders of oxidative stress. Expert Opin Ther Targets 16: 167-178, 2012.

14. Nakata R, Takahashi $\mathrm{S}$ and Inoue $\mathrm{H}$ : Recent advances in the study on resveratrol. Biol Pharm Bull 35: 273-279, 2012.

15. Gruber J, Tang SY and Halliwell B: Evidence for a trade-off between survival and fitness caused by resveratrol treatment of Caenorhabditis elegans. Ann NY Acad Sci 1100: 530-542, 2007.

16. Pasinetti GM, Wang J, Marambaud P, Ferruzzi M, Gregor P, Knable LA and Ho L: Neuroprotective and metabolic effects of resveratrol: Therapeutic implications for Huntington's disease and other neurodegenerative disorders. Exp Neurol 232: 1-6, 2011.

17. Brasnyó P, Molnár GA, Mohás M, Markó L, Laczy B, Cseh J, Mikolás E, Szijártó IA, Mérei A, Halmai R, Mészáros LG, et al: Resveratrol improves insulin sensitivity, reduces oxidative stress and activates the Akt pathway in type 2 diabetic patients. Br J Nutr 106: 383-389, 2011.

18. Bradamante S, Barenghi L and Villa A: Cardiovascular protective effects of resveratrol. Cardiovasc Drug Rev 22: 169-188, 2004.

19. Pulsinelli WA, Brierley JB and Plum F: Temporal profile of neuronal damage in a model of transient forebrain ischemia. Ann Neurol 11: 491-498, 1982.

20. Nikonenko AG, Radenovic L, Andjus PR and Skibo GG: Structural features of ischemic damage in the hippocampus. Anat Rec (Hoboken) 292: 1914-1921, 2009.

21. Li H, Zhang Y, Yu Y, Li B, Chen Y, Wu H, Wang J, Li J, Xiong X, He Q, Tian J, et al: Systemic revealing pharmacological signalling pathway networks in the hippocampus of ischaemia-reperfusion rats treated with baicalin. Evid Based Complement Alternat Med 2013: 630723, 2013.

22. Li J, Feng L, Xing Y, Wang Y, Du L, Xu C, Cao J, Wang Q, Fan S, Liu Q and Fan F: Radioprotective and Antioxidant effect of Resveratrol in Hippocampus by activating SIRT1. Int J Mol Sci 15: 5928-5939, 2014

23. Kang Y, Jung WY,Lee H, Lee E, Kim A and Kim BH: Expression of SIRT1 and DBC1 in gastric adenocarcinoma. Korean J Pathol 46: 523-531, 2012.

24. Matsushita T, Sasaki H, Takayama K, Ishida K, Matsumoto T, Kubo S, Matsuzaki T, Nishida K, Kurosaka M and Kuroda R: The overexpression of SIRT1 inhibited osteoarthritic gene expression changes induced by interleukin-1 $\beta$ in human chondrocytes. J Orthop Res 31: 531-537, 2013.

25. Chen F, Xu C, Du L, Wang Y, Cao J, Fu Y, Guo Y, Liu Q and Fan F: Tat-SmacN7 induces radiosensitization in cancer cells through the activation of caspases and induction of apoptosis. Int J Oncol 42: 985-992, 2013.

26. Fu Y, Wang Y, Du L, Xu C, Cao J, Fan T, Liu J, Su F, Fan S, Liu Q and Fan F. Resveratrol inhibits ionising irradiation-induced inflammation in MSCs by activating SIRT1 and limiting NLRP-3 inflammasome activation. Int J Mol Sci 14: 14105-14118, 2013.

27. Kitada M and Koya D: SIRT1 in Type 2 Diabetes: Mechanisms and therapeutic potential. Diabetes Metab J 37: 315-325, 2013.

28. Bernstein BE, Meissner A and Lander ES: The mammalian epigenome. Cell 128: 669-681, 2007.

29. Vaquero A, Scher M, Erdjument-Bromage H, Tempst P, Serrano L and Reinberg D: SIRT1 regulates the histone methyl-transferase SUV39H1 during heterochromatin formation. Nature 450: 440-444, 2007.

30. Nadtochiy SM, Redman E, Rahman I and Brookes PS: Lysine deacetylation in ischaemic preconditioning: The role of SIRT1. Cardiovasc Res 89: 643-649, 2011.

31. Shin SM, Cho IJ and Kim SG: Resveratrol protects mitochondria against oxidative stress through AMP-activated protein kinase-mediated glycogen synthase kinase-3beta inhibition downstream of poly (ADP-ribose) polymerase-LKB1 pathway. Mol Pharmacol 76: 884-895, 2009

32. Ramadori G, Lee CE, Bookout AL, Lee S, Williams KW, Anderson J, Elmquist JK and Coppari R: Brain SIRT1: anatomical distribution and regulation byenergy availability. $\mathrm{J}$ Neurosci 28: 9989-9996, 2008.

33. Tanno M, Sakamoto J, Miura T, Shimamoto K and Horio Y: Nucleocytoplasmic shuttling of the $\mathrm{NAD}^{+}$-dependent histone deacetylase SIRT1. J Biol Chem 282: 6823-6832, 2007.

34. Wassmann S, Wassmann K and Nickenig G: Modulation of oxidant and antioxidant enzyme expression and function in vascular cells. Hypertension 44: 381-386, 2004. 
35. Lu X, Ma L, Ruan L, Kong Y, Mou H, Zhang Z, Wang Z, Wang JM and Le Y: Resveratrol differentially modulates inflammatory responses of microglia and astrocytes. J Neuroinflammation 7: 46, 2010.

36. Terashvili M, Pratt PF, Gebremedhin D, Narayanan J and Harder DR: Reactive oxygen species cerebral autoregulation in health and disease. Pediatr Clin North Am 53: 1029-1037, 2006.

37. Zhang F, Liu J and Shi JS: Anti-inflammatory activities of resveratrol in the brain: Role of resveratrol in microglial activation. Eur J Pharmacol 636: 1-7, 2010.

38. Hu Y, Liu J, Wang J and Liu Q: The controversial links among calorie restriction, SIRT1 and resveratrol. Free Radic Bio Med 51: 250-256, 2011.

39. Nisoli E, Tonello C, Cardile A, Cozzi V, Bracale R, Tedesco L, Falcone S, Valerio A, Cantoni O, Clementi E, Moncada S, et al: Calorie restriction promotes mitochondrial biogenesis byinducing the expression of eNOS. Science 310: 314-317, 2005
40. Lagouge M, Argmann C, Gerhart-Hines Z, Meziane H, Lerin C, Daussin F, Messadeq N, Milne J, Lambert P, Elliott P, Geny B, et al: Resveratrol improves mitochondrial function and protects against metabolic disease by activating SIRT1 and PGC-1alpha. Cell 127: 1109-1122, 2006.

41. Csiszar A, Labinskyy N, Pinto JT, Ballabh P, Zhang H, Losonczy G, Pearson K, de Cabo R, Pacher P, Zhang C, et al: Resveratrol induces mitochondrial biogenesis in endothelial cells. Am J Physiol Heart Circ Physiol 297: H13-H20, 2009.

42. Pearson KJ, Baur JA, Lewis KN, Peshkin L, Price NL, Labinskyy N, et al: Resveratrol delays age-related deterioration and mimics transcriptional aspects of dietary restriction without extending life span. Cell Metab 8: 157-168, 2008.Lu X, Ma L. Ruan L, Kong Y, Mou H, Zhang Z, Wang Z, Wang JM and Le Y: Resveratrol differentially modulates inflammatory responses of microglia and astrocytes. J Neuroinflammation 7: 46, 2010. 